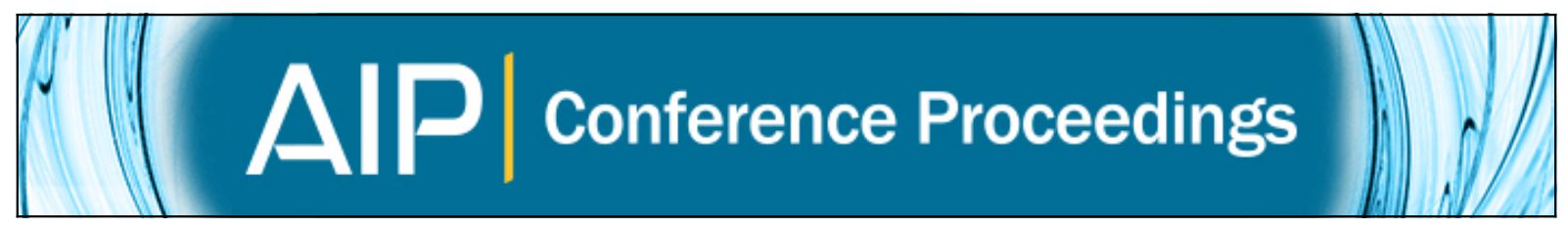

\title{
Mineralized polymer composites as biogenic bone substitute material
}

Rushita Shah, Nabanita Saha, Takeshi Kitano, and Petr Saha

Citation: AIP Conference Proceedings 1664, 070012 (2015); doi: 10.1063/1.4918447

View online: http://dx.doi.org/10.1063/1.4918447

View Table of Contents: http://scitation.aip.org/content/aip/proceeding/aipcp/1664?ver=pdfcov

Published by the AIP Publishing

Articles you may be interested in

Mössbauer study of biogenic formation processes of iron minerals

AIP Conf. Proc. 1489, 95 (2012); 10.1063/1.4759477

Improved accuracy of cortical bone mineralization measured by polychromatic microcomputed tomography using a novel high mineral density composite calibration phantom

Med. Phys. 37, 5138 (2010); 10.1118/1.3480507

Bone-composition imaging using coherent-scatter computed tomography: Assessing bone health beyond bone mineral density

Med. Phys. 33, 904 (2006); 10.1118/1.2179151

Determination of dose enhancement in cortical bone substitute material for electron beams

Med. Phys. 18, 324 (1991); 10.1118/1.596679

Comparison of absorbed dose in bone substitute material and water using ionization measurements

Med. Phys. 16, 81 (1989); 10.1118/1.596407 


\title{
Mineralized Polymer Composites as Biogenic Bone Substitute Material
}

\author{
Rushita Shah, Nabanita Saha*, Takeshi Kitano, Petr Saha \\ Centre of Polymer Systems, University Institute, Tomas Bata University in Zlin \\ Nad Ovcirnou 3685, 76001 Zlin, Czech Republic \\ *Contact e-mail ID: nabanita@,ft.utb.cz
}

\begin{abstract}
Mineralized polymer composites (MPC) are recognized as potential fillers of bone defects. Though bioceramics exhibits quite a good bone-bonding and vascularization, it is considered to be too stiff and brittle for using alone. Thus, the use of polymer scaffold instead of bioceramics has several advantages including combining the osteoconductivity and bonebonding potential of the inorganic phase with the porosity and interconnectivity of the threedimensional construction. Aiming the advantages of ceramic-polymer composite scaffolds, the calcium carbonate $\left(\mathrm{CaCO}_{3}\right)$ based biomineralized scaffold was prepared, where the PVP-CMC hydrogel was used as an extracellular matrix. This paper is reported about the morphology, swelling trend (in physiological solution) and viscoelastic behavior of ( $90 \mathrm{~min}$ mineralized) MPC. The dry MPC are off-white, coarse in texture, comparatively less flexible than the original PVP-CMC based hydrogel film, and the deposition of granular structures on the surface of the hydrogel film confirms about the development of biomineralized scaffold/polymer composites. Irrespective of thickness, the dry MPC shows higher values of swelling ratio within $30 \mathrm{~min}$, which varies between 200-250 approximately. The dynamic viscoelastic nature of freshly prepared MPC was investigated applying $1 \%$ and $10 \%$ strain. At higher strain the viscoelastic moduli ( $G$ ' and G") show significant change, and the nature of MPC turns from elastic to viscous. Based on the observed basic properties, the MPC (calcite based polymer composites) can be recommended for the treatment of adyanamic bone disorder.
\end{abstract}

Key words: Adyanamic bone disorder, biomineralization, calcium carbonate, PVP-CMC hydrogel, polymer composite scaffold.

PACS: $81.07 \mathrm{Pr}, 81.05 \mathrm{Rm}, 87.85 \mathrm{jf}, 83.85 \mathrm{Cg}$.

\section{INTRODUCTION}

Biomimetic materials inspired in biology are of interest in these days for material scientist in several biomedical fields. The properties of hydrogels as unique biomaterials came in focus since $20^{\text {th }}$ century with the development of soft contact lenses [1]. In the swollen state, they are soft and rubbery, thus resembling a living tissue [2]. Ongoing investigations on natural and synthetic hydrogels have established their potential use in several biomedical applications such as drug delivery systems, soft contact lenses, implants, bone-tissue regeneration, dental plaques etc. [35]. However, natural based polymeric materials, polysaccharides form the prominent member due to its non-toxicity, biocompatibility, availability in large variety of composition and properties [6-9]. Biominerals formed by organic/inorganic composites resemble the materials obtained from geological processes [10]. Increasing interest has been shown in polymer composites as potential fillers of bone defects. The preparation of composite itself is a biomimetic approach [11].

Proceedings of PPS-30

AIP Conf. Proc. 1664, 070012-1-070012-5; doi: 10.1063/1.4918447

(C) 2015 AIP Publishing LLC $978-0-7354-1309-2 / \$ 30.00$

070012-1 
Bone, for example is made up of collagen, the principal organic component, hydroxyapatite, the inorganic mineral component, water and small amounts of other organic phases [11,12].The composites made from organic-inorganic hybrid material using biocompatible polymeric material in form of hydrogels and also biominerals like calcite or calcium phosphates show promising trend in the field of mineralization [12-14]. Mineralization within the hydrogel can be modified by various ways in presence of particular substrate. Furthermore, convenient scaffolds prepared by mineralization can better mimic the native microenvironment [12].

The significance in the present study lies in the fact that these hydrogels after mineralization can be utilized in biomedical applications like adynamic bone disorder. For accomplishing this task, firstly the mineralized hydrogel is prepared by liquid diffusion technique [3-5, 14]. PVP-CMC hydrogel was prepared by solvent casting technique, and then its dried film was used as a matrix for biomineralization for $90 \mathrm{~min}$. Finally, the developed calcite filled polymeric composite material designated as mineralized polymeric composites (MPC), has been characterized following the established techniques. This paper presents about morphology, swelling trend and rheological behaviour of MPC.

\section{EXPERIMENTAL}

The dry PVP-CMC hydrogel [6-9] were used as a matrix, and the solutions of $\mathrm{CaCl}_{2} .2 \mathrm{H}_{2} \mathrm{O}(14.7 \%, \mathrm{pH}=8.4)$ and $\mathrm{Na}_{2} \mathrm{CO}_{3}(10.5 \%, \mathrm{pH}=7.4)$ were used for mineralization applying the liquid diffusion techniques. The PVP-CMC hydrogel was soaked in $\mathrm{CaCl}_{2} \cdot 2 \mathrm{H}_{2} \mathrm{O}$ and $\mathrm{Na}_{2} \mathrm{CO}_{3}$ solutions simultaneously after regular interval of 15 mins. The $90 \mathrm{~min}$ mineralized hydrogel were used for characterization, thus the samples washed in distilled water and incubated for freeze drying as well as air drying at room temperature respectively. The freeze dried MPC were used for morphological investigation, air dried MPC were used to evaluate the swelling capacity in physiological solution, and fresh/ before dry MPC were used for rheological measurements.

The morphology observation of calcite filled and unfilled PVP-CMC hydrogels was carried out using a scanning electron microscope (SEM) (VEGA II LMU (TESCAN)), which is operated in the high-vacuum/ secondary electron imaging mode at an accelerating voltage of 5$20 \mathrm{kV}$. The images were taken at the magnification of 100x-10kx.

The swelling studies of the MPC were carried out in the physiological solution $(\mathrm{pH}=7.5$ and temperature $=37^{\circ} \mathrm{C}$ ). The swollen gels in the physiological solution were taken out at regular time intervals, wiped superficially, weighed, and placed in the same bath. In this way, the cycle of swelling was repeated from 0-300 mins in the regular time interval of 30 mins each. The percentage of swelling was obtained using the following expressions where, $\mathrm{W}_{\mathrm{s}}$ is for the swollen gel in physiological solution and $\mathrm{W}_{\mathrm{d}}$ is the weight of dried MPC [6].

$$
\text { Swelling } \%=[(W s-W d)+(W d)] * 100
$$

The Rheological properties (in the form of dynamic viscoelastic nature) of the MPC were investigated by using a parallel plate rheometer (ARES; Rhemetrics Scientifics, USA) testing with a "TA Orchestrator" software package. A $25 \mathrm{~mm}$ diameter parallel plate measuring geometry with a gap of about 2-3 mm was used, employed at strain amplitude (1\%) to maintain the measurement range within the linear viscoelastic region. Dynamic frequency sweep test were carried out at the temperature of $28^{\circ} \mathrm{C}$ to observe the storage $\left(\mathrm{G}^{\prime}\right)$ and loss $\left(\mathrm{G}^{\prime \prime}\right)$ moduli, and complex viscosity $\left(\eta^{*}\right)$ as a function of a wide range of angular frequency $(\omega: 0.1-100 \mathrm{rad} / \mathrm{s})$. 


\section{RESULTS AND DISCUSSION}

\section{Morphology of MPC}

Figure 1 shows the surface analysis of matrix (i.e. PVP-CMC hydrogel) and MPC (i.e. calcite filled PVP-CMC hydrogel matrix). It can be seen from the Figure 1(a) that several pores are exhibited within the 3-dimensional cross-linked structure of pure PVP-CMC hydrogel matrix. After liquid $\left(\mathrm{CaCl}_{2}\right.$ and $\left.\mathrm{Na}_{2} \mathrm{CO}_{3}\right)$ diffusion mineralization, conducted up to $90 \mathrm{~min}$, the pores of hydrogel matrix first filled up with calcium ions and finally developed $\mathrm{CaCO}_{3}$ crystal structures which can be visible clearly from Figure 1(b). In mesocystal theory, it is explained about the aggregation and development of crystals of calcite within the hydrogel matrix [14]. The crystals obtained on PVP-CMC hydrogel matrix are granular in shape and with increase in mineralization time, increases the size of crystals.
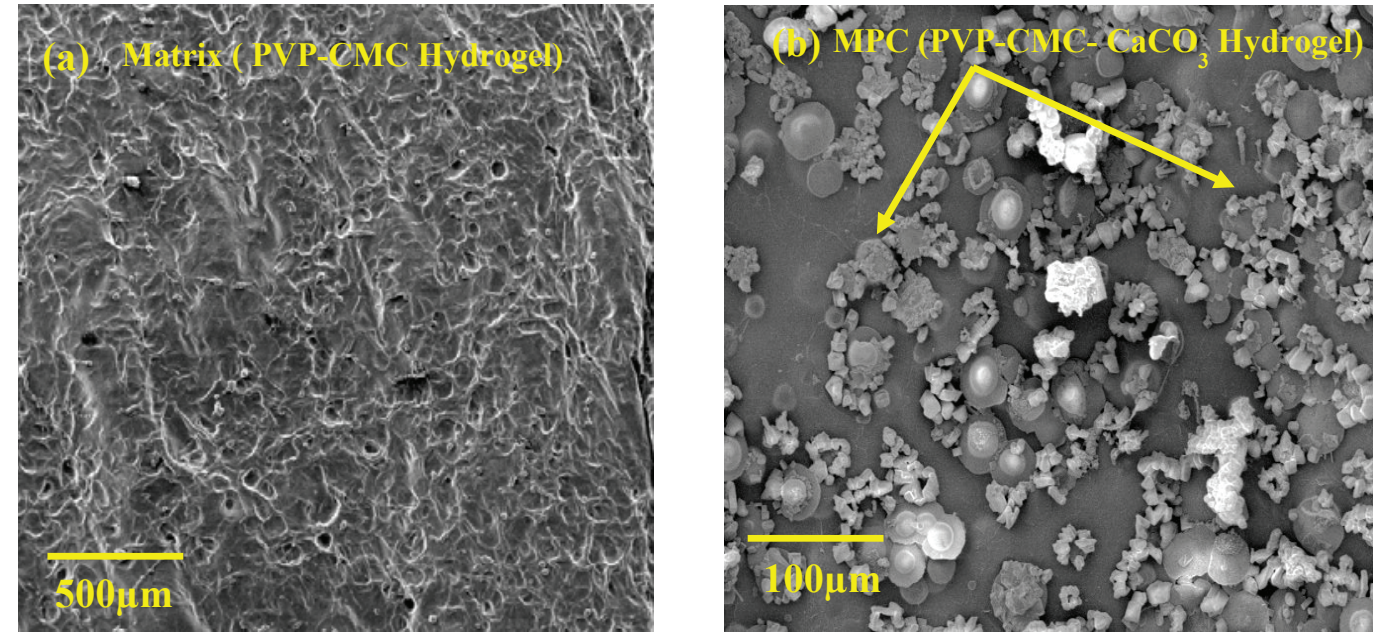

FIGURE 1: SEM Images of (a) Matrix (PVP-CMC hydrogel, surface image before mineralization) and (b) MPC (PVP-CMC-CaCO 3 hydrogel, surface image after 90 min mineralized)

\section{Swelling of MPC}

The swelling capacity of MPC was measured in physiological solution $(0.9 \% \mathrm{NaCl})$ as body fluid, blood plasma contains about $90-92 \%$ of water. The osmotic pressure of the physiological solution is equivalent to human blood tissue [15]. Four different thicknesses $(100,200,300,400 \mu \mathrm{m})$ of mineralized hydrogels i.e. MPC were taken for investigation of the swelling property. It can be observed from Figure 2 that irrespective of thickness, in each case the initial rate of swelling is high, and the values of swelling ratio vary between 200-250. The swelling ratio of calcite filled polymer composite increases with time until $180 \mathrm{~min}$ but after that the values are getting more or less constant. This indicates that the degree of swelling value reaches to equilibrium condition after around $180 \mathrm{~min}$. 


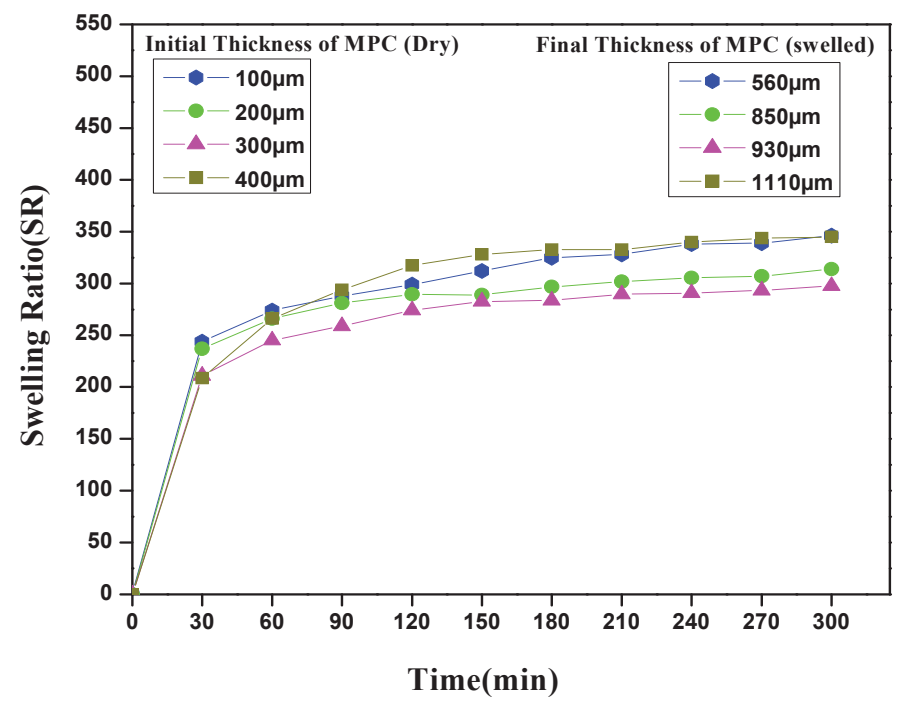

FIGURE 2: Swelling behaviour of MPC in physiological solution

\section{Viscoelastic Behaviour of MPC}

Figure 3 shows the viscoelastic properties of MPC (mineralized with $\mathrm{CaCO}_{3}$ for 90 min). It can be observed through the figure that the values of $G^{\prime}$ is higher and $\omega$ independent in MPC at $1 \%$ strain compared to G', which indicates the elastic behaviour is dominant to viscous nature. Moreover, the linear curve confirms the solidity of the material over wide range of deformation rate. But, when the strain increases up to $10 \%$, the values of G' decrease to the level of G" which indicates the conversion of elastic nature of composite material to viscous nature. Moreover, the complex viscosity $\eta^{*}$ of MPC decreases linearly with increasing of $\omega$ in doublelogarithmic coordinates, and also decreases with increasing of strain.

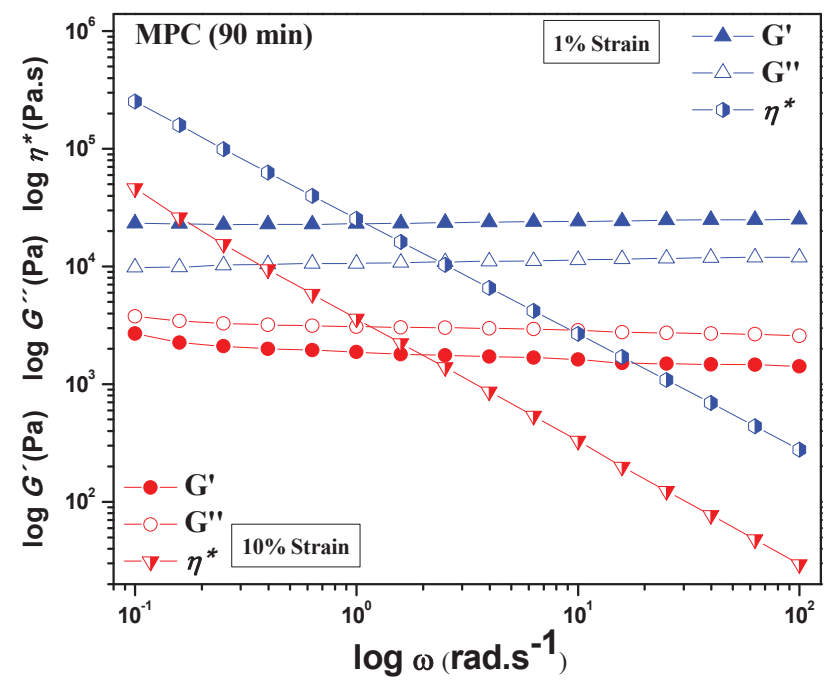

FIGURE 3: Effect of angular frequency $(\omega)$ at $1 \%$ and $10 \%$ strain on storage modulus (G', filled symbol), loss modulus (G”, non-filled symbol) and complex viscosity ( $\eta^{*}$, half-filled symbol) for mineralized polymer composite (90 min). 


\section{CONCLUSION}

The liquid diffusion technique used for preparation of mineralized polymer composite (MPC).This MPC biomaterial can be utilized in biomedical applications like adyanamic bone disorder. The morphological image confirms the deposition of $\mathrm{CaCO}_{3}$ into the matrix. The swelling study of MPC confirmed that irrespective of thickness the swelling ratio (SR) values are initially high until $30 \mathrm{~min}$ and thereafter gradually rises and get then more or less constant after $180 \mathrm{~min}$. Further, the rheological properties of the MPC explained the elastic and viscous nature of the calcite filled biomaterial at low and high strain over wide range of deformation rate.

\section{ACKNOWLEDGMENTS}

The authors are thankful for the support of Research and Development for Innovation Operational Programme co-funded by the European Regional Development Fund (ERDF) and national budget of the Czech Republic within the framework of the Centre of Polymer Systems project (reg. number: CZ.1.05/2.1.00/03.0111) and the support of the "Education for Competitiveness" Operational Programme co-funded by the European Social Fund (ESF) and the national budget of the Czech Republic, within the "Advanced Theoretical and Experimental Studies of Polymer Systems" project (reg. number: CZ.1.07/2.3.00/20.0104). This work was also conducted within the framework of COST Action MP1301 "New Generation Biomimetic and Customized Implants for Bone Engineering" - www.cost.eu. Authors are also thankful to Internal Grant Agency (IGA/FT/2014/015) of Tomas Bata University in Zlin for providing financial support to carry out this research.

\section{REFERENCES}

1. A.A. Ashley and L. S. Nair, Biomed. Mater. 7, 1-13 (2012)

2. S.K.Bajpai, J.Appl.Poly.Sci, 80, 2782-2789 (2001)

3. R. Shah, N.Saha, T. Kitano, P.Saha, J.Appl.Poly.Sci,131(10) (2014) DOI: 10.1002/APP.40237

4. N.Saha, R. Shah, R.Vyroubal, T. Kitano, P.Saha, Novel Trends in Rheology V Book Series: American Institute of Physics 1526, 2013, pp. $292-300$.

5. N.Saha, R.Vyroubal, R. Shah, T. Kitano, P.Saha, Novel Trends in Rheology V Book Series: American Institute of Physics 1526, 2013, pp. 301-309.

6. N.Roy, N.Saha, T.Kitano, P.Saha, J. Appl. Polym. Sci. 117, 1703-1710 (2010).

7. N.Saha, A.Saarai., N.Roy., T.Kitano and P.Saha. JBNB 2, 85-90 (2011).

8. P.Saha, N.Saha, N.Roy, Czech Republic, Patent No: CZ 302405B6 (4 May 2011).

9. N.Roy, N.Saha, T.Kitano, P.Saha, Carbohydrate Polymers 89, 346-353, (2012).

10. L.A.Estroff and I. Cohen, Nature Materials, 10, 810-811(2011).

11. H.E. Davis and J.K. Leach, Hybrid and Composite Biomaterials in Tissue Engineering, California, Ed. N Ashammakhi, 1999, pp.1-26.

12. N.H.Munro and K.M.McGrath, Bioinsp. Biomime. and Nanobiomaterials, 1, 26-37 (2011).

13. T.Nonoyama, H.Ogasawara, M. Tanaka, M. Higuchi, T. Kinoshita, Soft Matter 8, 11531-11536 (2012).

14. M. W. Rauch, M. Dressler, H. Scheel, D. V. Opdenbosch and C. Zollfrank, Eur. J. Inorg. Chem, 5192-5198 (2012).

15. N.Roy, N.Saha, T.Kitano, and P.Saha, Soft Materials, 8(2), 130-148, (2010). 